\title{
IMPROVING THE LINEARITY OF FERRITE LOADED CAVITIES USING FEEDBACK
}

\author{
J. Dey ${ }^{\dagger}$, J. Steimel, FNAL*, Batavia, IL 60510, USA
}

\section{Abstract}

A simple beam loading compensation system was installed for the Fermilab Main Injector Coalescing Cavities. This paper describes the design and implementation of the feedback system. These modifications improved the linear dynamic range of operation of the ferrite loaded cavity.

\section{INTRODUCTION}

In the Fermilab Main Injector there are five ferrite loaded Coalescing Cavities [1] that resonate at $2.5 \mathrm{MHz}$. Each Cavity has 34 rings of Philips 4M2 ferrite and each ring is $500 \mathrm{~mm}$ OD X $200 \mathrm{~mm}$ ID X $25.4 \mathrm{~mm}$ thick. The unloaded Q of a cavity is $125 \pm 5$ and the gap voltage is $15 \mathrm{kV}$ during Coalescing. The shunt impedance is $50 \mathrm{k} \Omega$ and when operated in the $12 \mathrm{kV}$ to $17 \mathrm{kV}$ range the shunt impedance decreases by approximately $10 \%$. Each cavity is powered by a Lambda RF Systems model PA5K-30LC, water cooled, solid-state linear $5 \mathrm{~kW}$ MOSFET amplifier.

With the new requirements of the Fermilab Recycler, an individual cavity will now have to operate down to $400 \mathrm{~V}$ to match the $2 \mathrm{kV}$ transfer requirements of the Main Injector to Recycler. In order to meet these new specifications a simple feedback system was implemented to linearize the response of an individual cavity.

\section{DIRECT RF FEEDBACK}

The fundamental purpose of direct RF feedback is to have the RF voltage at the cavity gap accurately follow the low level radio frequency (LLRF) drive to the system. This is accomplished by combining the cavity gap monitor signal with the LLRF drive to the system. When the proper phase delay is given to the returning cavity gap monitor signal, a negative feedback system is created. In determining the proper phase delay one must not violate the Nyquist stability criteria for the open loop response of the system [2].

\section{IMPLEMENTING RF FEEDBACK}

The original feedforward system only consisted of the 5 $\mathrm{kW}$ Lambda RF Systems amplifier and the $2.5 \mathrm{MHz}$ ferrite loaded coalescing cavity shown in Figure 2.

In order to do the open loop design, a Hewlett Packard 8753E Network Analyzer (NA) was used. The 8753E was configured as shown in Figure 1. Test Port Power was set to $-30 \mathrm{dBm}$ and two $16 \mathrm{~ns}$ cables were attached and calibrated out. The diagram in Figure 2 was converted to open loop mode by terminating the LLRF port of the PD20-50 splitter with $50 \Omega$ s and hooking the other input splitter port to Port 1 of the $8753 \mathrm{E}$ using one of the calibrated out $16 \mathrm{~ns}$ cable. The remaining Port 2 calibrated out 16 ns cable was connected to the delay line. The electrical delay line feature of the NA was then used to determine the final length of delay line which was 36 ns.

The 3.25 MHz Low-Pass Filter shown in Figure 3 was used to dampen the mode at $4.9 \mathrm{MHz}$ as seen in Figure 1 coming back from the cavity. This was done because the 4.9 $\mathrm{MHz}$ mode seen in S21 Real would go above one violating the Nyquist stability criteria with just moderate gain.

The final gain was done by adjusting the attenuators before the delay line and before the non-inverting MiniCircuits ZHL-3A amplifier. The Figure 1 channel 2 marker shows an overall loop gain of 5.3319 at 2.537 MHz. Also, Figure 1 shows that the Real part was never allowed to exceed 0.4 over the frequency range of 1 to 11 MHz.

After doing the open loop measurement, the NA with its two $16 \mathrm{~ns}$ cables were removed from the system and the loop was closed as shown in Figure 2.

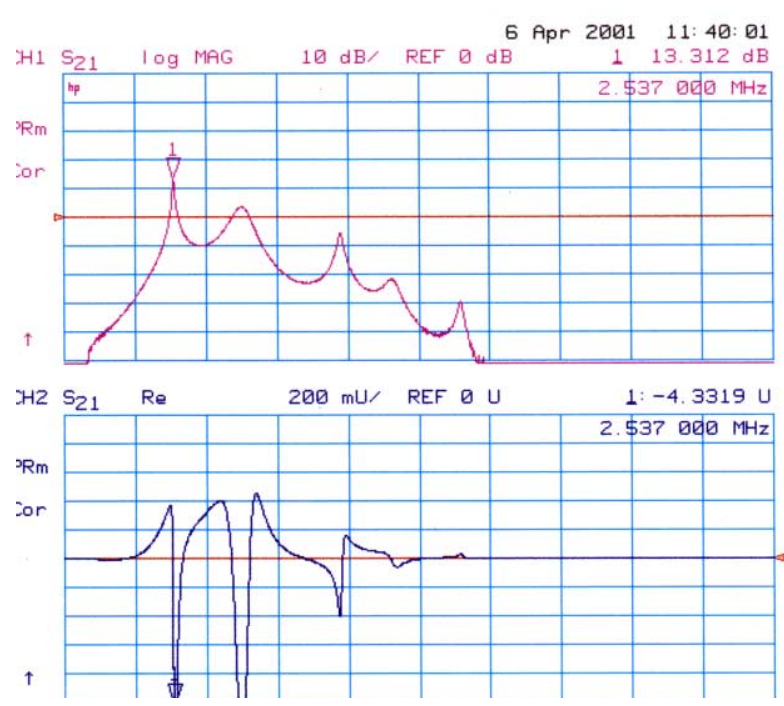

Figure 1: Coalescing Cavity Open Loop Response with zero electrical delay. 


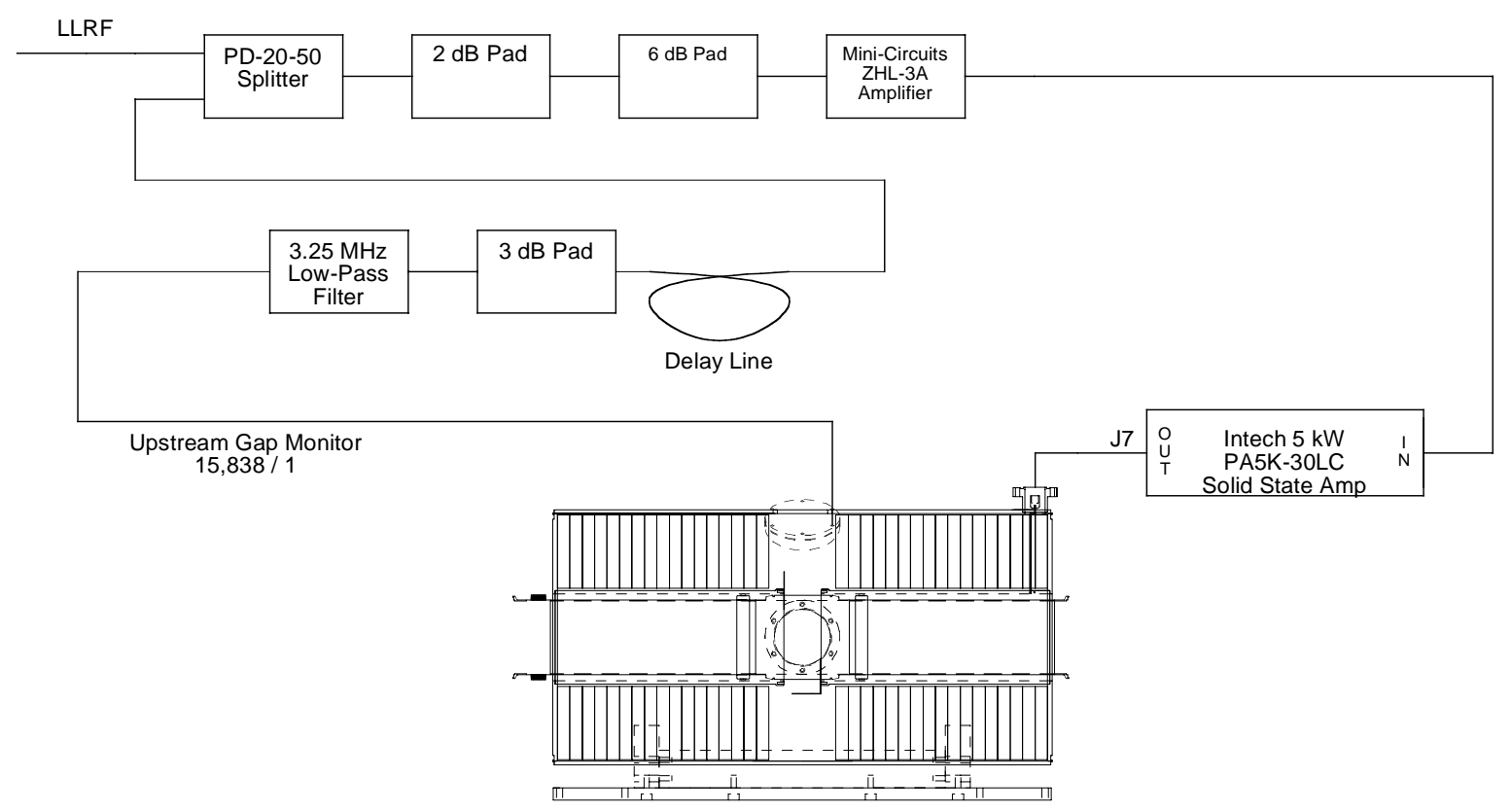

Figure 2: Feedback Diagram of the Main Injector $2.5 \mathrm{MHz}$ Coalescing Cavities.
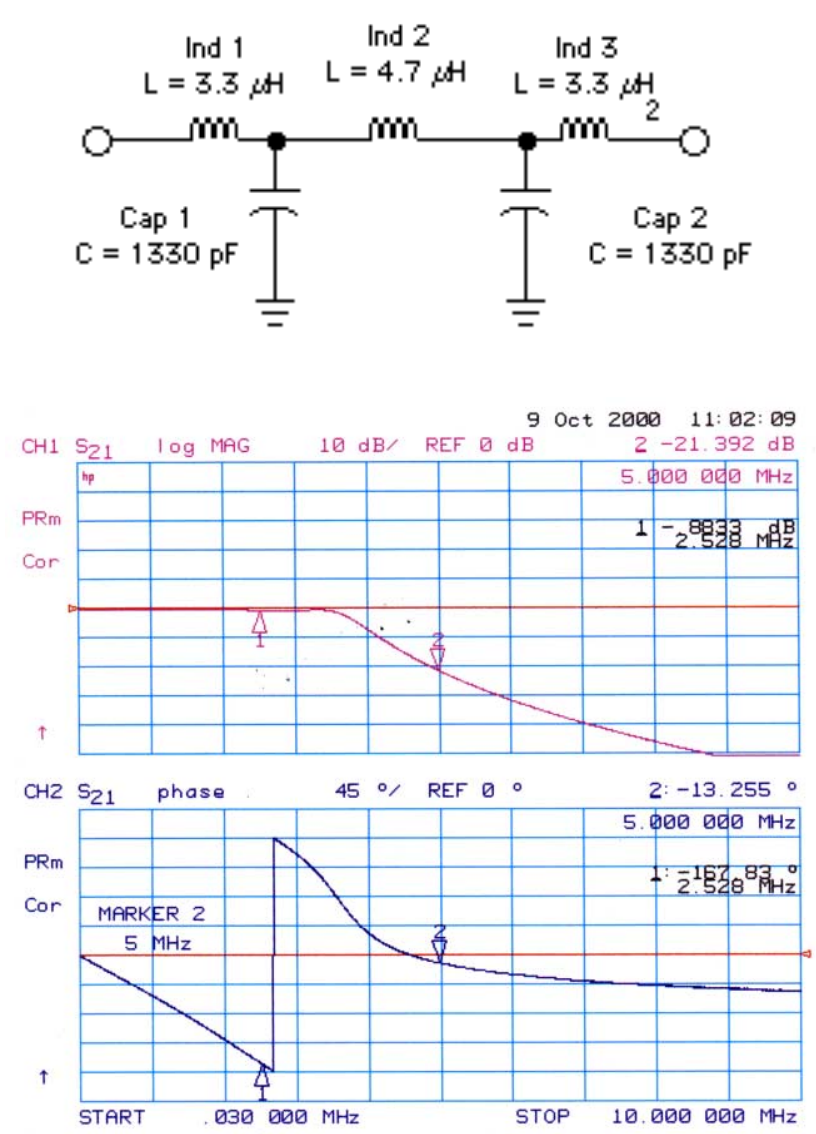

Figure 3: 3.25 MHz Low-Pass Filter and its Response.

\section{RESULTS}

Figure 4 shows the lowering of the $\mathrm{Q}$ of the Coalescing Cavity with feedback. The measurement was done with the NA Port 1 driving the LLRF port of the splitter and Port 2 looking at the cavity's response at the Downstream Gap Monitor. Again, the Test Port Power was set to -30 $\mathrm{dBm}$. Channel 1 shows the trace with feedback $\mathrm{Q}$ (magenta) to be 19.122 while the trace without feedback $\mathrm{Q}$ (green) was 84.862. The Q was reduced by over a factor of four with RF Feedback. Note also how the phase change flattened out with feedback (blue) versus without feedback (red) in Figure 4.

Figure 5 is the exact same setup as Figure 4 but now the frequency was held constant at $2.514 \mathrm{MHz}$ and Port 1 of the NA was swept from $-15 \mathrm{dBm}$ to $10 \mathrm{dBm}$ in power. This corresponds to sweeping the Gap Voltage from 600 $\mathrm{V}$ to $11.33 \mathrm{kV}$. $2.514 \mathrm{MHz}$ is the $8 \mathrm{GeV}$ injection frequency Main Injector to Recycler. The Channel 1 plot shows how linear the ferrite loaded cavity response becomes with feedback (magenta) versus without feedback (green). It is visually noticeable how the ferrite cavity has been linearized. Channel 2 in Figure 5 shows how the phase only traveled 16.33 degrees between Markers 1 and 2 with feedback (blue). The phase traveled 48.57 degrees without feedback (red). 

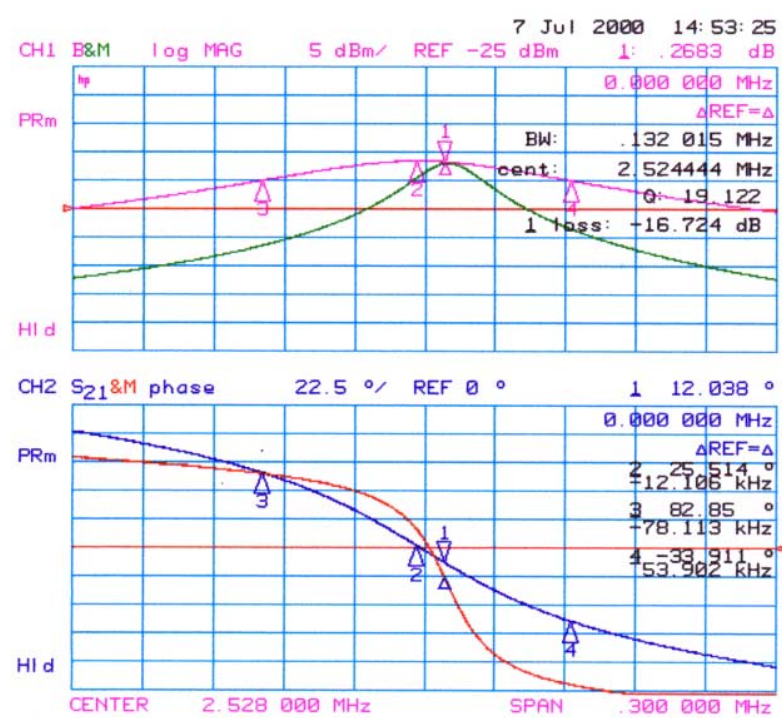

Figure 4: Coalescing Cavity Response with (magenta) and without (green) Feedback.
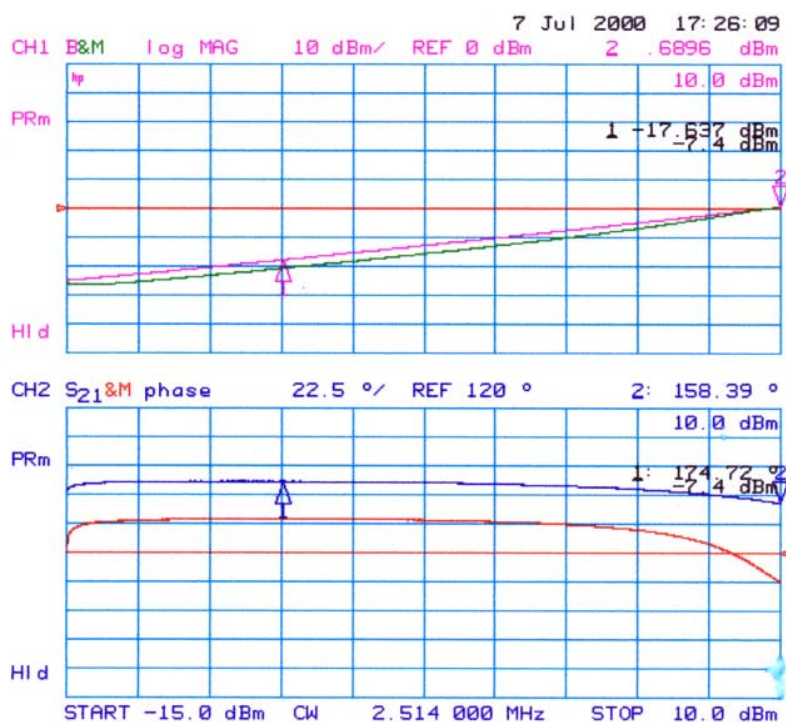

Figure 5: Coalescing Cavity Power Sweep with (magenta) and without (green) feedback.

\section{REFERENCES}

[1] J. Dey, I. Kourbanis, D. Wildman, "A New RF System for Bunch Coalescing in the Fermilab Main Ring,” 1995 PAC, p. 1672, Dallas, May 1995.

[2] J. Dey, J. Steimel, J. Reid, "Narrowband Beam Loading Compensation in the Fermilab Main Injector Accelerating Cavities," these proceedings. 